\title{
Coping Measurement and the State Effect of Depression and Anxiety in Psychiatric Outpatients
}

\author{
Toru Uehara $^{a}$ Kaoru Sakado ${ }^{\text {b }}$ Tetsuya Sato $^{c}$ Ryu Takizawad \\ aDepartment of Neuropsychiatry, University of Gunma Faculty of Medicine, Maebashi, bDepartment of Psychiatry, \\ Niigata University School of Medicine, Niigata, J apan; cPsychiatrische Klinik und Poliklinik, \\ Ludwig-Maximilians-Universität München, Deutschland; dUniversity of Gunma School of Medicine, Maebashi, J apan
}

\section{Key Words}

Depression · Anxiety · Trait · State · Coping Inventory for Stressful Situations

\begin{abstract}
The relationship between coping styles and mental health has received considerable attention, but the state effects on coping measures in a clinical sample are not well known. This study investigated changes in scores on the Coping Inventory for Stressful Situations between two treatment phases (acute and remitted phase) in 49 outpatients with major depression or anxiety disorders. Task-oriented coping changed significantly between the treatment phases in both depressive and anxious patients, as analyzed by two-way multivariate analysis of variance. Results from repeated measures of multivariate analysis of covariance indicated that task-oriented coping was influenced by depression and emotionoriented coping was influenced by anxiety. Avoidanceoriented coping did not change significantly over time in either depressive or anxiety disorders controlled for depressive and anxiety symptoms. The results of this study suggest that depressive or anxiety symptoms and treatment phase affect coping measurement.
\end{abstract}

Copyright @2002 S. Karger AG, Basel

\section{Introduction}

The relationship between coping styles and mental disorders such as depression and anxiety has received considerable attention in a number of studies [1-6]. These studies indicated that depressed or anxious individuals use more emotion-oriented and fewer task-oriented coping strategies than nonsymptomatic persons. These studies were conducted using either normal subjects or a student sample. There are some reports of coping characteristics in a clinically diagnosed sample. Cronkite et al. [7] reported that a poor-outcome group of depressed adults was less easygoing and relied more on an avoidance coping style than remitted and normal control groups. Ravindran et al. [8] confirmed that emotion-focused coping was correlated with the severity of the depressive affect. They suggested a relationship between depression and emotionor avoidance-oriented coping styles.

It remains unclear whether coping with stress is a state or trait measure. Coping behaviors have sometimes been defined as trait and sometimes as state variables [9, 10]. Which type of variable applies is one of the principal questions of coping theory. We need to examine the influences of state effects on coping measures such as depression and anxiety. One purpose of this study was to

Toru Uehara, MD, PhD

Instructor/Assistant Professor, Department of Neuropsychiatry

University of Gunma Faculty of Medicine

3-39-22 Showa-machi, Maebashi, Gunma 371-8511 (Japan)

Tel. +81 27220 8187, Fax +81 27220 8192, E-Mail toruaki@med.gunma-u.ac.jp 
Table 1. Mean scores for coping measures and clinical evaluations for the diagnostic group at baseline (T1) and remission (T2)

\begin{tabular}{|c|c|c|c|c|c|c|c|c|c|}
\hline & \multicolumn{2}{|c|}{ Major depression } & \multicolumn{2}{|c|}{ Anxiety disorders } & \multicolumn{2}{|c|}{ Simple main effect, F } & \multirow{2}{*}{$\begin{array}{l}\text { Diagnostic group } \\
\text { by time interaction } \\
\mathrm{F}(1,45)\end{array}$} & \multicolumn{2}{|c|}{ Time by covariate, $\mathrm{F}$} \\
\hline & $\mathrm{T} 1$ & $\mathrm{~T} 2$ & $\mathrm{~T} 1$ & $\mathrm{~T} 2$ & time & group & & HRSD & SAS \\
\hline \multicolumn{10}{|l|}{ CISS } \\
\hline T scale & 48.7 & 50.7 & 49.5 & 51.5 & $10.99 * *$ & 0.65 & 3.58 & $10.81 * *$ & 0.01 \\
\hline E scale & 48.9 & 44.4 & 43.4 & 42.9 & 0.84 & 2.52 & 3.15 & 0.5 & $4.11^{*}$ \\
\hline A scale & 43.2 & 40.7 & 42.2 & 41.2 & 0.07 & 0 & 2.43 & 0.31 & 0.13 \\
\hline \multicolumn{10}{|c|}{ Clinical evaluations } \\
\hline HRSD & 18.3 & 3.4 & 9.2 & 3.6 & & & & & \\
\hline SAS & 49.4 & 32.6 & 42.2 & 33.4 & & & & & \\
\hline
\end{tabular}

Repeated-measure MANOVA: $* * \mathrm{p}<0.01$. MANCOVA F ratio for differences over time controlled for change in symptoms: $* \mathrm{p}<0.05$, $* * \mathrm{p}<0.01$.

clarify the interactions between coping and changes in symptoms in patients with major depression or anxiety disorders using the Coping Inventory for Stressful Situations (CISS) [11], a reliable coping measure.

\section{Subjects and Methods}

Study Participants

The subjects in this study were 36 outpatients with major depressive disorder and 13 outpatients with anxiety disorders ( 9 with panic disorders and 4 with generalized anxiety disorders) diagnosed using the Diagnostic and Statistical Manual of Mental Disorders, Fourth Edition [12] at Niigata City General Hospital, Japan. Inclusion criteria were as follows: (1) patients were in remission from episodes after 4 months of regular treatment [for depressive patients, remission was defined as a score of below 5 on the 17-item Hamilton Rating Scale for Depression (HRSD) [13] over at least a 4-week period, and for patients with anxiety, a score of below 39 on the Self-Rating Anxiety Scale (SAS) [14] and nearly remitted anxiety symptoms]; (2) patients showed no severe physical complications, psychotic features, dysthymia or manic episodes, and (3) the age of the patients ranged from 18 to 65 years. The mean age of the 49 subjects was 38.5 years (SD 12.2 years). Thirty subjects $(61.2 \%)$ were female. Subjects averaged 2.4 previous episodes; the mean length of the current depressive/anxiety episode prior to treatment was 2.8 months. We included full responders to ordinary treatment (pharmacotherapy using antidepressants and/or anxiolytics and supportive psychotherapy). Informed consent was obtained from all subjects.

\section{Materials and Procedure}

At baseline (the time of diagnosis) and 4 months later, the HRSD, the Beck Depression Inventory (BDI) [15], the SAS, the Global Assessment of Functioning (GAF) [12] and the CISS were administered to the patients $(n=49)$. The mean scores and SDs at baseline were 15.5 and 7.0 on the HRSD, 22.8 and 9.8 on the BDI, 47.0 and 8.9 on the SAS and 59.3 and 5.8 on the GAF, respectively. At remission, the mean scores and SDs were 3.9 and 3.0 on the HRSD, 4.1 and 4.9 on the BDI, 34.4 and 8.0 on the SAS and 71.8 and 4.8 on the GAF, respectively. The CISS is a self-rating coping measure developed by Endler and Parker [11] and consists of three coping dimensions: task oriented $(\mathrm{T})$, emotion oriented $(\mathrm{E})$ and avoidance oriented (A). The reliability and validity of the Japanese version of the CISS has been confirmed by Furukawa et al. [16].

Changes in scores for each subscale of the CISS were calculated by subtracting the score at baseline from the remission score. Changes in scores for the HRSD, BDI, SAS and GAF were calculated in the same manner (scores at remission minus scores at baseline). Because the changes in scores were statistically dependent on the initial score (the score at baseline), we calculated residual scores for all dimensions, and these were adjusted for the regression of the respective change in scores on the initial score. A repeated measure of multivariate analysis of variance (MANOVA) was used to test whether scores on the CISS changed over time. In order to control for symptomatology, depression severity (residual change in scores on the HRSD) and anxiety severity (residual changes in scores on the SAS) were entered in as covariates (multivariate analysis of covariance; MANCOVA).

\section{Results}

Comparisons of the mean scores of the three CISS subscales between baseline and remission using repeated MANOVA are presented in table 1. Time (baseline or remission) was the within-subject variable and group (major depression or anxiety disorder) was the between-subject variable. The $\mathrm{T}$ score changed significantly over time $(\mathrm{F}=10.99, \mathrm{p}<0.01$, d.f. $=1,45)$, but the simple main effect of group and group by time interaction were not 
significant. The E and A scores did not change significantly with time or by group. We next considered whether the changes in test scores differed according to changes in depression or anxiety. After controlling for depression between two occasions of testing by separating out the residual change in the HRSD scores from changes in the coping measures, the time by depression interaction was significant on the $\mathrm{T}$ scale $(\mathrm{F}=10.81, \mathrm{p}<0.01$, d.f. $=1,43)$. After controlling for anxiety (the SAS score), the time by anxiety interaction was significant on the $\mathrm{E}$ scale $(\mathrm{F}=$ $4.11, \mathrm{p}<0.05$, d.f. $=1,43)$.

\section{Discussion}

There are many studies which show that assessment of personality is influenced by clinical symptoms such as depression and anxiety [17]. Individual coping constructs are closely related to personality, and thus state effects on coping measures must be resolved. Many previous reports have suggested that emotion-focused coping is strongly related to psychopathology (e.g. relationships with the BDI score, neuroticism and psychopathological scales of the Minnesota Multiphasic Personality Inventory) [18, 19] and have shown that emotion-focused coping is highly correlated with depression and anxiety in both nonclinical [20] and clinical samples [4]. It is important to clarify the stability of coping measures and interactions with symptomatology using longitudinal observations.

Our results on a clinical sample also indicated that $T$ and $E$ scales of the CISS were associated with symptoms and the treatment process. By multivariate analysis, the $T$ scale of the CISS changed significantly over time in both the depressive and anxious patients, and it was especially affected by changes in depressive symptoms, as analyzed by MANCOVA. We must consider that changes in taskoriented coping may be influenced by the state effect, and that this may have a strong relation to depressive symptoms. On the other hand, the E and A scales did not show significant change over time by MANOVA. Only anxiety symptoms were correlated with the E scale by MANCOVA. Emotion-oriented coping might not change in a clinical sample compared with a normal sample possibly because patients with depression or anxiety disorders may naturally show higher scores on this scale. The interaction between the E scale and anxiety was in agreement with that found in numerous other studies. In addition, the A scale could be generally considered as a trait measure in both depression and anxiety disorders because the main effect over time was not significant.

The present study indicates that the $\mathrm{T}$ scale changes by treatment phase, and that depressive symptoms have a great influence on the $\mathrm{T}$ score. The $\mathrm{E}$ score seems to be affected by anxiety, as previous studies have suggested for normal subjects. However, avoidance-oriented coping, which includes social support seeking, might show more trait characteristics. Overall, we need to consider that coping styles tend to adopt state effects with symptoms of depression and anxiety. However, in a clinical sample, changes in this coping by treatment phase were limited. The major limitation of the present study lies in the inclusion of a heterogeneous sample with mixed diagnoses of depressive and anxiety disorders. In the present study, we wanted to know the state effect of symptoms on coping measures, but we must mention that there would be differences between the groups in coping styles. Since there were no comparisons with normal subjects, we do not know whether the changes that might occur with the treatment reflect 'normalization' of coping, or whether they continue to reflect pathological coping. To clarify the interaction between coping and psychopathological symptoms or psychiatric disorders, we need to compare responders to nonresponders.

\section{References}

1 Coyne JC, Aldwin C, Lazarus RS: Depression and coping in stressful episodes. J Abnorm Psychol 1981;90:439-447.

2 Paker GB, Brown LB: Coping behaviors that mediate between life events and depression. Arch Gen Psychiatry 1982;39:1386-1391.

3 Billings AG, Moos RH: Coping, stress, and social resources among adults with unipolar depression. J Pers Soc Psychol 1984;46:877891.
4 Folkman S, Lazarus RS: Stress processes and depressive symptomatology. J Abnorm Psychol 1986;95:107-113.

5 Endler NS, Parker JDA: Multidimensional assessment of coping: A critical evaluation. J Pers Soc Psychol 1990;58:844-854.

6 Rohde P, Lewinsohn PM, Tilson M, Seeley JR: Dimensionality of coping and its relation to depression. J Pesrs Soc Psychol 1990;58:499511.
7 Cronkite RC, Moos RH, Twohey J, Cohen C, Swindle R Jr: Life circumstances and personal resources as predictors of the ten-year course of depression. Am J Community Psychol 1998; 26:255-280.

8 Ravindran AV, Griffiths J, Merali Z, Anisman $\mathrm{H}$ : Primary dysthymia: A study of several psychosocial, endocrine and immune correlates. J Affect Disord 1996;40:73-84. 
9 Lazarus RS: Coping theory and research. Past, present, and future. Psychosom Med 1993;55: 234-247.

10 Livneh H, Livneh CL, Maron S, Kaplan J: A multidimensional approach to the study of the structure of coping with stress. J Psychol 1996; 130:501-512.

11 Endler NS, Parker JDA: Coping Inventory for Stressful Situations (CISS): Manual. Toronto, Multi-Health Systems, 1990.

12 American Psychiatric Association: Diagnostic and Statistical Manual of Mental Disorders, ed 4. Washington, American Psychiatric Association, 1994.
13 Hamilton M: A rating scale for depression. J Neurol Neurosurg Psychiatry 1960;23:56-62.

14 Zung WWW: A rating instrument for anxiety disorders. Psychosomatics 1971;12:371-379.

15 Beck AT, Ward CH, Mendelson ML: An inventory for measuring depression. Arch Gen Psychiatry 1961;4:561-571.

16 Furukawa T, Suzuki-Moor A, Saito Y, Hamanaka T: Reliability and validity of the Japanese version of the coping inventory for stressful situations (CISS): A contribution to the cross-cultural studies of coping (in Japanese). Seishin Shinkeigaku Zasshi 1993:95:602-620.
17 Boyce P, Hadzi-Pavlovic D, Parker G, Brodaty H, Hickie I, Mitchell P, Wilhelm K: Depressive type and state effects on personality measures. Acta Psychiatrica Scand 1990;81:197-200.

18 Endler NS, Parker JDA: State and trait anxiety, depression, and coping. Aust J Psychol 1990; 42:207-220.

19 Endler NS, Parker JDA, Butcher JN: A factor analytic study of coping styles and MMPI-2 content scales. J Clin Psychol 1993;49:523527.

20 Rosenberg SJ, Peterson RA, Hayes JR: Coping behaviors among depressed and nondepressed medical inpatients. J Psychosom Res 1987;31: 653-658. 